\title{
ALTERNATIVE APPROACH FOR A TOTAL INTEGRATED SECONDARY INSTALLATION IN MV SUBSTATIONS COVERING ALL POSSIBLE AND REQUIRED FUNCTIONS
}

\author{
M.J.M. VAN RIET*, F.L. BALDINGER+, W.M. VAN BUIJTENEN\#, F.T.J. VAN ERP*, F. VOLBERDA*, F. PROVOOST^ \\ *Nuon Tecno, The Netherlands, \\ +Locamation, The Netherlands, \\ \#Phase to Phase, The Netherlands, \\ $\wedge$ Eindhoven University of Technology, The Netherlands \\ Maarten.van.Riet@Nuon.com
}

\section{INTRODUCTION}

A simple, flexible and cheap automation concept for $M V$ substations has been developed. The concept consists of a fully integrated system, containing accurate data acquisition, metering, protection and control. It is in line with IEC 61850. The basics of the system were fully type tested during 2004. In 2005 it will be implemented in two substations in order to proof the data acquisition in combination with several central monitoring systems within the company. The next step will be the integration with protection and control in 2006. As from 2007 this system will be used during substation refurbishment.

\section{BACKGROUND}

The fundamental difference in lifetime of existing primary equipment and nowadays applied secondary technology feeds a growing concern of the grid operators. A concern of the manageability of future refurbishment projects of secondary installations has always to be seen in perspective of the continuous decrease of the technical staff. The liberalization of the energy market leads to an increasing demand for more detailed information of the distribution network performance and behaviour. The required information is most often not retrievable from the existing secondary installation, so new function boxes are needed. Unfortunately the directly related benefits arising from this information are quite often not justifying the investment. This is the main reason, that projects on medium voltage substation automation are hardly executed and even cancelled during the last years.

The outgoing feeders of HV/MV substations are mostly the border between different market players. Therefore this point is the ideal point for metering, Power Quality registrations and monitoring loads and currents.

For the coming years NUON has to refurbish more than 200 HV/MV substations. This has to be done for reasonable prices. The currently available solutions consist of separate boxes for every function, each with it's own wiring, programming language and manufacture specific properties. This will often result in inflexible complex and expensive solutions. Especially for system operations.

NUON already realized an integrated pilot project in substation Zaltbommel [1]. This concept however was still far too complex and too expensive. Especially the engineering and installation of the new system in the old environment was extremely time consuming.

\section{THE NEW SYSTEM}

\section{General}

According to NUON the key to success will be a total new developed approach based on low initial investment and simple and inexpensive future extension. The simple interface between primary and secondary equipment (figure 2) must be future proof so new required functionality can easily be installed without major changing of the basis. For a MV installation the functionality will cover: local and remote control, protection, disturbance recording, fault localization, metering of class $0.2 \mathrm{~S}$, power quality monitoring functions, primary equipment diagnostics and all other necessary secondary functionality. Signal converters will send/receive all data to a central system where all required functionality is implemented. The communication interface between the signal converters and the centralized system as well as the signal converters themselves are specially designed on cost effectiveness, simplicity and long life time.

Although the integrated secondary installation system concept is designed on price effectiveness and simplicity it fully caters for the high requirements resulting from the functions like protection, metering and power quality monitoring (figure 3). The concept is in line with the IEC 61850 standard on the data model and high level interfaces.

This new concept has been developed in narrow cooperation between a utility, and two relatively small and therefore flexible software and hardware developing companies all having many years of experience with grid and substation concepts.

\section{Description of the system}

The starting point for the system design, application and operation in substations is based on sense and simplicity. Emphasis is given to all aspects of engineering, installation, commissioning, testing, operation, maintenance, long life of applied components and future extendibility.

The system architecture features two key parts, i.e. the primary process interfacing and a separated application processing.

Fundamental in the system architecture is the interface between the primary equipment and secondary system. The design of this interface is of major importance for both the life cycle cost of the total secondary installation as well as its 
functional performance during its long technical life.

One of the design goals for the process interface devices is the technical and functional life which should be in the range of the life time of the primary equipment where it is connected too. Due to the expected shorter technical life of the central processing devices, where the application functionality is executing, these devices should be easy replaceable.

Next to these requirements the system must contain a minimal number of different physical devices. Our slogan is: “everything which isn't there doesn't need to be bought, engineered, tested, maintained, understood and last but not least can't fail either".

Simplicity of the system architecture and its physical implementation is of major importance for all related aspects.

\section{Architecture}

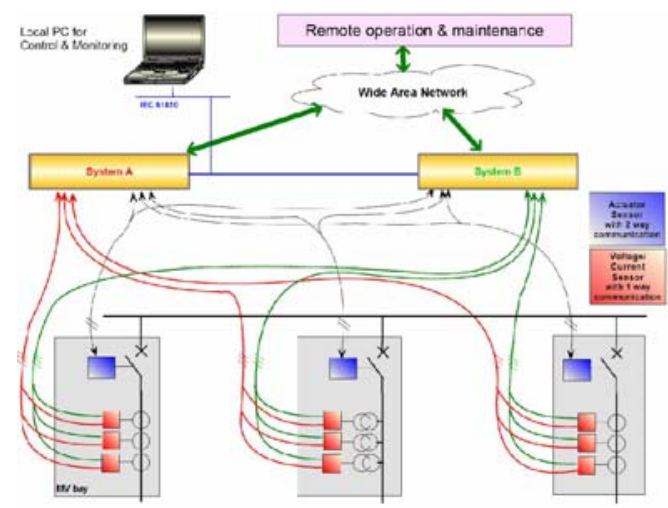

Figure 1: The schematically new system architecture

The architecture shown in figure 1 is simple as explained before and consists of two groups of devices. We have interface devices and application processing devices. The interface device group covers the devices for primary process interfacing like voltage \& current transformers, actuator position sensors for circuit breakers etc. and interface devices for legacy secondary equipment. The processing device forms the computing heart of the system. In a MV application only one (or two for redundancy) central processing device is required. This processing device will run all possibly required application functionality and possibly interfaces with other function boxes for external applications by using the IEC61850 standardized serial interfaces.

\section{Data acquisition}

A main part of the cost of secondary systems is the interfacing with the primary installation. If this interface can have the same life time, as least functional, as the primary components future upgrade/refurbishments will become simple and cheap. The process interface will digitize the primary signals into digital information. The long life of the process interface necessitates a long term view on what possibly can be required in the future to make sure that the digitized signals contain all information. Accurate and very high speed sampling is one of them.

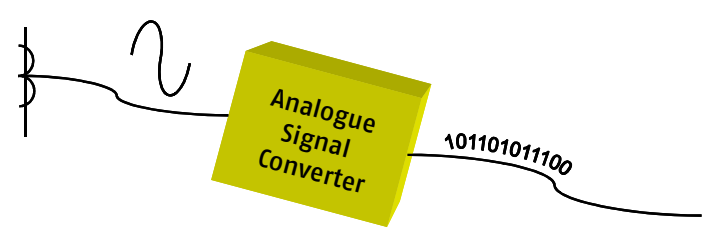

Figure 2: The analogue signals are converted into bit trains with future proof information content

\section{Central Processing Devices}

The central system is a "one-box" device but with a multiprocessor environment and a flexible amount of fiber optic interfaces for sensors, actuators, serial intelligent electronic devices and Ethernet based devices. On the central level all functionality is implemented like control, overcurrent protection, revenue metering class $0.2 \mathrm{~s}$, power quality monitoring and all other applications as long as they can be written in software. The object modelling and external communication interfaces are in line with the IEC61850.

The real-time operating system contains an embedded web server to enable a graphical user interface bases on common web technology. The PC only needs a web browser and optionally a secure connection mechanism is available with encryption (not IEC61850). The graphics are based on HTML and SVG as industry standards.

System maintenance and handling of new application functions can be done online locally or remote by secure connections on TCP/IP. Application functions as well as new executables can be added, started, stopped without redoing the whole application configuration.

Online embedded version control is implemented to track changes and enable roll back. On top of this the "layered" file system opens a lot of engineering opportunities. It can separate changes from approved configurations, but also enables easy online simulation and testing possibilities. The proof by design of the simulation methods will save significant time during on site commissioning work and future modifications. High speed sensor data can be simulated by software on separate processor boards without interfering the performance of the existing processors.

The proof by design and transparency of the internal system operation eases the job of the application engineers and return the full understanding with what they are doing and want to achieve. Sense and simplicity on all levels.

\section{INTEGRATION OF PROTECTION AND CONTROL}

If "off-the-shelve" protection \& control functions from other suppliers are preferred the standardized IEC61850 interface will bring the solution in sight. The central processing unit will resample the high speed, high precision samples of the data acquisition part to the lower speed IEC61850-9-2 process bus samples. These samples will be used by the third party 
protection \& control devices which will run their own application according to their specs. The possible trip messages will be given directly to the actuators on basis of the IEC61850-8-1 station bus.

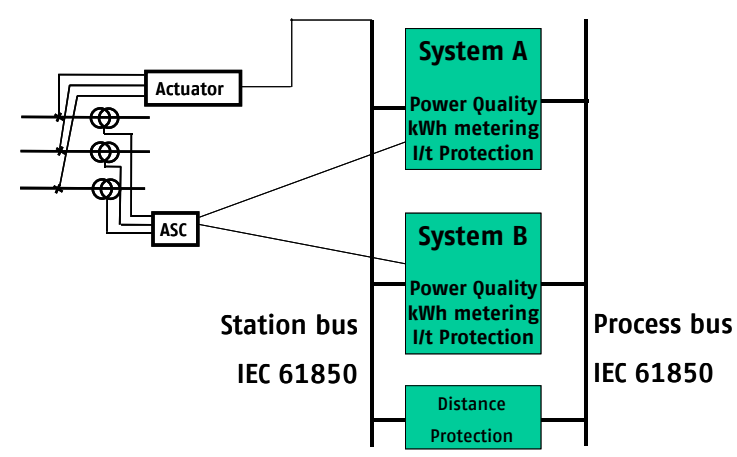

\section{FEASIBILITY}

Figure 3: The new integrated secondary installation

The feasibility of the new proposed system is examined in a detailed study. In this study questions were asked to departments within Nuon related to substation automation. Grid operators, asset managers and engineers where involved. Asset managers and operators need more information and tools to manage and operate the complex situation in the MV grid that exists nowadays. Asset managers require information on historical loads for expansion scenarios. Operators are interested in actual loads to support remote switching activities. Dedicated software functions in the central device transform the data from the sensors into useful information for these end-users.

Because of the flexibility of the centralized processing software the new automation system can keep up with the growing information need without large investments compared to conventional systems.

The financial analysis showed that the new automation system will cost $25 \%$ of a conventional system. Most of the cost reduction is established by decreasing the effort on engineering, installation, configuration and testing. Besides the vast reduction of initial costs, maintenance and update costs are also being kept low. Only the central processing device and its software might be upgraded when new or different functionality is wanted or the applied processors become obsolete.

\section{RESULTS OF TESTING THE SYSTEM}

\section{Metering}

The system is able to use data from the protection coil of MV current transformers for metering. The tests were performed according to IEC 62053-22;2003-01. We proved that the accuracy in the whole range (1\% up till $120 \%$ of nominal current) was more than 10 times better than the requirements of the Dutch revenue metering regulations.

\section{Power Quality}

The tests were performed according to IEC 61000-4-7 (Testing and measurement techniques- General guide on harmonics and interharmonics measurements and instrumentation, for power supply systems and equipment connected thereto). The system is able to measure harmonics up to the $50^{\text {th }}$ and all other Power Quality aspects stated in IEC 50160, like voltage dips, sags, swells, flicker, etc.

\section{IMPLEMENTATION AND PILOT}

In 2005 the system will be implemented in two substation as a pilot. The aim of this pilot is to proof the accuracy of the different parts of the measuring system. The measuring results are transferred from the substation towards different departments within Nuon, where involved people must be able to simply obtain and process the data in order to get proper management and operational information. The system is build in such a way, that data is stored local in the substation itself. The information can be uploaded via the Nuon communication network, the so called Cuprum project [2].

From the intelligent MV fault localization pilot in Zaltbommel [1, 3] we learned quite a lot about the best way of triggering for starting registration of voltage and current waveforms during faults in different types of networks. Two and three phase faults normally are not a problem. Correct triggering of single phase to ground faults is more complex. Self extinguishing faults must be distinguished from real faults switched off by remote protection devices. The lessons learned will be implemented in the new system

With the new system simple sophisticated protection is feasible. This will result in a protection system that is fast enough to minimize impacts of dips and retains its selectivity with respect to remote protection in the network.

\section{OTHER RELEVANT PILOTS}

To reach the final goal several pilots are or will be realized. Examples are:

- Intelligent Medium Voltage fault localization [3]

- Estimation of equalising currents on the moment of coupling MV grids fed by two different HV/MV substations based on on-line phasor measurement and smart grid calculations.

- Experiments based on $\mathrm{PhD}$ works in the Dutch innovative R\&D program on intelligent networks $[4,5]$.

\section{CONCLUSIONS}

A simple cheap system for operation, protection, control intelligent fault location and data acquisition has been developed. This system will be implemented and tested in 2005 and 2006 in several substations and several pilot projects. The total functional scope of the system will be in operation in 2007. With this solution the refurbishment of substation automation will be simple, fast and economically feasible. The open structure facilitates simple future extensions. 


\section{REFERENCES}

[1] M.J.M. van Riet, F. Provoost, F. van Erp, 2003, "New features during refurbishment of substations", CIRED 2003

[2] M.J.M. van Riet, F. Provoost, , F. van Erp, 2004, "New intelligent features during refurbishment of substations", IEE symposium on Developments in Power Systems Protections.

[3] P.M. van Oirsouw, F. Provoost, 2003, "Fault location in an medium voltage network ", CIRED 2003

[4] Reza M, Schavemaker P.H., Kling W.L.,Sluis L. van der, 2003, " A research program on intelligent power systems : self controlling and self adapting power systems equipped to deal with the structural changes in the generation and the way of consumption ", CIRED 2003

[5] Homepage for Dutch innovative research program on intelligent networks www.intelligentpowergrids.nl 